\title{
Magnetoimpedance in soft magnetic amorphous and nanostructured wires
}

M. L. Sánchez, V. M. Prida, J. D. Santos, J. Olivera, T. Sánchez, J. García, M. J. Pérez \& B. Hernando

Applied Physics A

Materials Science \& Processing

ISSN 0947-8396

Volume 104

Number 1

Appl. Phys. A (2011)

104:433-445

DOI 10.1007/s00339-011-6245-

z

\section{Applied Physics A}

Materials

Science \& Processing

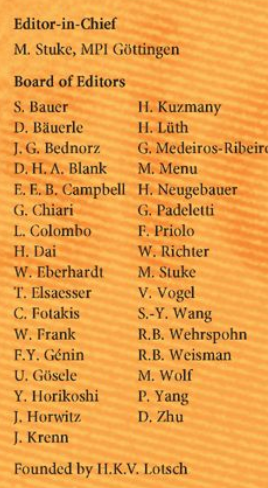

2) Springer
Special Issue: "Synchrotron Radiation in Art and Archeology"

Guest Editors: I. Reiche, B. Kanngießer, W. Eberhardt

Editorial

G. Chiari, R. Giustetto, J. Druzik, E. Doehne, G. Ricchiardi

Pre-columbian nanotechno
of the maya blue pigment

R. Arletti, L. Cartechini, R. Rinaldi, S. Giovannini,

W. Kockelmann, A. Cardarelli
Texture analysis of bronze age axes by neutron
diffraction

V. Kokka, A. Psarrou, V. Konstantinou
Computational models for pigments analysis

A. Lluveras, S. Boularand, I. Roqué, M. Cotte,

P. Giraldez, M. Vendrell-Saz
Weathering of gilding decorations investigated by S development and distribution of calcium oxalates in the
case of Sant Benet de Bages (Barcelona, Spain)

T. Broekmans, A. Adriens, E. Pantos

Insights into the production lechnology of
north-Mesopotamian Bronze Age pottery

M. Manso, M. Costa, M.L. Carvalho

Comparison of elemental content on modern and ancient papers by EDXRF

M. Gil, M.L. Carvalho, A. Seruya, I. Ribeiro, 1. Queralt,
A.E. Candeias, I. Miräo A.E. Candeias, T.Mirào
Limewashing paintings in Alentejo urban heritage: Limewashing paintings in Alentejo urban heritage:
pigment characterization and differentiation by WDXRI
and XRD

M. Sánchez del Río, A. Gutiérrez-León, G.R. Castro, J. Rubio-Zuazo, C. Solis, R. Sánchere-Hernánder, 
Your article is protected by copyright and all rights are held exclusively by SpringerVerlag. This e-offprint is for personal use only and shall not be self-archived in electronic repositories. If you wish to self-archive your work, please use the accepted author's version for posting to your own website or your institution's repository. You may further deposit the accepted author's version on a funder's repository at a funder's request, provided it is not made publicly available until 12 months after publication. 


\title{
Magnetoimpedance in soft magnetic amorphous and nanostructured wires
}

\author{
M.L. Sánchez • V.M. Prida • J.D. Santos • J. Olivera • \\ T. Sánchez • J. García • M.J. Pérez • B. Hernando
}

Received: 23 June 2010 / Accepted: 22 December 2010 / Published online: 18 January 2011

(C) Springer-Verlag 2011

\begin{abstract}
The surface impedance tensor approach has been used to review the impedance response in a variety of amorphous and nanocrystallized wires. An experimental study on the torsion annealing effect on the magnetoimpedance (MI) behaviour for positive and negative magnetostriction amorphous wires of $\mathrm{FeSiB}$ and $\mathrm{CoSiB}$ compositions, respectively, has been carried out. Moreover, the influence of the onset nanocrystallization on the MI behaviour in Finemet-type alloys, with particular attention focussed on the case that the wires are annealed under applied torsional stress, is also presented. The analysis of the MI and torsion-impedance (TI) effects allows us to compare the different magnetic characteristics observed in a variety of wires. Special attention is paid to new results of the off-diagonal MI huge responses of these ferromagnetic samples, which are also a very useful source of information on the magnetic properties of the wires.
\end{abstract}

\section{Introduction}

The magnetoimpedance (MI) effect has become an attractive subject in magnetic materials research, due to its enhanced possibilities as technological applications and also as a research tool [1-7]. The MI effect consists on a large variation of impedance when a magnetic bias field is applied to a ferromagnetic conductor when an ac current is flowing through it. The MI effect depends on the ac drive current (frequency

M.L. Sánchez $(\varangle)$ V V.M. Prida · J.D. Santos · J. Olivera

T. Sánchez · J. García · M.J. Pérez · B. Hernando

Departamento de Física, Universidad de Oviedo, 33007 Oviedo,

Spain

e-mail: mlsr@uniovi.es

Fax: +34-985103324 and amplitude) and on the bias longitudinal field through the skin depth and magnetic permeability, respectively. This effect has been used in many applications so far. It is possible to tailor materials to achieve the desired application, in order to get adequate MI responses.

The magnetic response of the sample can also be influenced by other parameters that characterize the sample, like the magnetic anisotropy [8], permeability or magnetostriction coefficient [10]. As a general rule, samples with compositions corresponding to very low or nearly zero magnetostriction coefficient can show large magneto impedance effect, but in the case of samples with larger magnetostriction, the tensional or torsional stresses can also improve their magnetic behaviour through magnetoelastic coupling [11]. A torsion-impedance (TI) effect can also be defined as due to the influence of a torsional stress, without the influence of any magnetic field. It consists of the variation of impedance due only to a torsional stress applied to the sample [10, 12$16]$.

The soft ferromagnetic wires, produced by rapid solidification from the melt, have become one of the most important developments during the last decade [17]. Due to the absence of a long-range crystalline structure, the shape and magnetoelastic anisotropies together with magnetostatic effects are the main source of anisotropy in soft ferromagnetic amorphous wires. The quenching process induces strong radial stresses and hence stress-induced anisotropy in the radial direction. Besides that, a magnetic anisotropy can be induced in amorphous wires by performing thermal treatment under a tensile or torsional stress, affecting their MI behaviour.

It is well known that the microstructure control of the samples greatly affects the magnetic behaviour of ferromagnetic materials, producing a magnetic softening of the sample, and increasing its magnetic permeability [18-20]. The 
nanocrystalline alloys obtained by devitrification of Fe-rich metallic glasses have very interesting magnetic properties, such as high saturation magnetization, vanishing macroscopic anisotropy, and high magnetic permeability, which make them suitable for exhibiting high MI responses [21].

A way of obtaining a helical induced anisotropy is to make a thermal annealing with the simultaneous application of a torsional stress to the sample. Thus, the measurement of the off-diagonal components of impedance can give information about the stress state of the sample, and its magnetoelastic response through the MI effect or the TI effects.

The off-diagonal MI has recently attracted large interest due to its advantages for its application in the development of magnetic sensors. This effect has shown a great potential of amorphous ferromagnetic wires, microwires and ribbons for microminiature magnetic field sensing applications. Their main advantages are the high response non hysteretic behaviour, low power consumption and very simple sensor scheme, which make them very promising materials for sensor technology [22-25].

MI and TI effects are studied in this review in ferromagnetic wires with different compositions, when submitted to the influence of torsional stresses in both cases, during the annealing treatment and during the measurement. Co-rich and Fe-rich wires have different signs of magnetostriction constant, therefore resulting in an opposite magnetic response. The influence of nanocrystallization onset is also reported for Fe-rich wires. The off-diagonal MI effect was also obtained for these wires and is reported in Sect. 3.7, showing large responses.

\section{Experimental details}

The amorphous wires have been obtained by the in-rotatingwater quenching technique. The magnetostriction coefficients were measured by the small angle magnetization rotation (SAMR) method [26]. The hysteresis loops were obtained by a conventional induction technique at $12 \mathrm{~Hz}$ and a maximum magnetic field up to 120 Oe. Magnetic domains have been observed by Bitter technique in a metallographic microscope. Ferrofluid nanoparticles were supplied by Ferrotec, Inc. USA, and domain observations were done under a constant magnetic field of $24 \mathrm{kAm}^{-1}$ perpendicularly applied to the wire axis to enhance the contrast.

In order to further improve the soft magnetic properties of the $\mathrm{Fe}_{73.5} \mathrm{Si}_{13.5} \mathrm{~B}_{9} \mathrm{Nb}_{3} \mathrm{Cu}_{1}$ alloy and obtain an optimum microstructure, amorphous wires of this composition have been annealed at different temperatures. The evolution of the magnetic parameters that have been obtained after heat treatments leads to an improvement in the impedance response for the nanocrystallized material. This alloy with a nanocrystalline grain structure exhibits excellent soft magnetic properties based on the two main phases of the microstructure by consisting of nanocrystalline ferromagnetic grains surrounded by a ferromagnetic amorphous matrix. Since the grain size (about 10-20 nm) of the $\alpha$ FeSi nanocrystalline phases is much smaller than the magnetic exchange correlation length, the magnetocrystalline anisotropy and the magnetostrictive coefficients are averaged over the many small grains, which can be accounted for by the random anisotropy model [20].

The amorphous $\mathrm{Fe}_{73.5} \mathrm{Si}_{13.5} \mathrm{~B}_{9} \mathrm{Nb}_{3} \mathrm{Cu}_{1}$ samples were cut in $20 \mathrm{~cm}$ long pieces in order to perform the annealing treatments. Some of them were annealed at $560^{\circ} \mathrm{C}$ during 20 minutes in a preheated furnace, under Ar atmosphere. Others were annealed in the same way, but with an applied torsional stress of $\pi / 20 \mathrm{rad} / \mathrm{cm}$, in order to induce a helical anisotropy. The wires have a $98 \mu \mathrm{m}$ diameter, and smaller parts of around $7.0-8.5 \mathrm{~cm}$ in length were cut from the central part of the annealed wires for magnetic measurements.

Another set of amorphous $\mathrm{Fe}_{73.5} \mathrm{Si}_{13.5} \mathrm{~B}_{9} \mathrm{Nb}_{3} \mathrm{Cu}_{1}$ were annealed in a conventional preheated furnace at temperatures ranging between $300^{\circ} \mathrm{C}$ and $560^{\circ} \mathrm{C}$ during 1 hour, under Argon atmosphere, in order to achieve the nanocrystalline state. These wires were $10 \mathrm{~cm}$ long and had a larger diameter, of $133 \mu \mathrm{m}$.

Another set of amorphous wires with nominal compositions $\mathrm{Fe}_{77.5} \mathrm{Si}_{7.5} \mathrm{~B}_{15}$ and $\mathrm{Co}_{72.5} \mathrm{Si}_{12.5} \mathrm{~B}_{15}$ were also studied. Diameters of the samples were $126 \mu \mathrm{m}$ and $120 \mu \mathrm{m}$, respectively. As-quenched samples of both compositions, were also cut in $20 \mathrm{~cm}$ long pieces for performing the thermal treatments. Samples were annealed at $350^{\circ} \mathrm{C}$ during 20 minutes in Ar atmosphere without magnetic field and simultaneously twisted with a torsional strain of $\pi / 20 \mathrm{rad} / \mathrm{cm}$ in the counter clock-wise sense, which was kept constant during the treatments. The annealing temperature was selected well below the corresponding one to the beginning of any crystallization process (above $400^{\circ} \mathrm{C}$ for both samples). The Curie points of the amorphous magnetic phases of $\mathrm{Fe}_{77.5} \mathrm{Si}_{7.5} \mathrm{~B}_{15}$ and $\mathrm{Co}_{72.5} \mathrm{Si}_{12.5} \mathrm{~B}_{15}$ wires were $440 \pm 10^{\circ} \mathrm{C}$ and $280 \pm 10^{\circ} \mathrm{C}$ respectively. The thermal treatment was carried out at a temperature below the Curie point in the case of the Fe-based wires and above the corresponding Curie point for the Co-based wires. Due to this reason, the induced anisotropy in this wire should only be brought about by the applied torsional stress. The nature of the torsion-induced magnetic anisotropy in the $\mathrm{Fe}_{77.5} \mathrm{Si}_{7.5} \mathrm{~B}_{15}$ wires would be a magnetoelastic one [27].

The MI effect consists on a large variation of impedance as a magnetic field is applied to a ferromagnetic sample. When an ac drive current is flowing through the sample, two components of the induced voltage can be measured, as a consequence of a cross-magnetization process.

The voltage response in the magnetic wires $\left(V_{z}, V_{\phi}\right)$ is related to the ac excitation current $I_{z}$ via the surface im- 


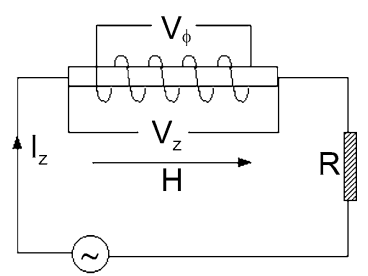

Fig. 1 Experimental set-up for the measurement of axial and off-diagonal magnetoimpedance in a ferromagnetic sample. The sample is connected in series with a resistor $R$, in order to maintain a constant amplitude of the ac drive current, $I_{z}$. Two measured voltages are obtained, one between the ends of the sample $V_{z}$, and another one by means of a pick-up coil, $V_{\phi}$. A bias magnetic field $H$ can be applied axially to the sample by means of a pair of Helmholtz coils

pedance tensor. This tensor establishes the relationship between the tangential electric field (which originates the voltage) and the tangential magnetic field (which determines the current) on the wire surface. The diagonal axis $\zeta_{z z}$ and offdiagonal $\zeta_{\phi z}$ components of the impedance tensor can be derived from the voltage $V_{z}$ between the wire ends for the conventional MI, or the voltage $V_{\phi}$ of the pick-up coil, respectively $[23,25]$ :

$\zeta_{z z}=\left(V_{z} / I_{z}\right)(2 \pi a / L)$

$\zeta_{\phi z}=\left(V_{\phi} / I_{z}\right)(1 / N)$

Being $L$ the wire length and $N$ is the number of turns of the pick-up coil. The components of the surface impedance tensor of the wire have been determined by measuring the induced voltages, $V_{z}$ and $V_{\phi}$, using a two-channel lock-in amplifier.

Figure 1 shows a scheme of the experimental set-up for the impedance tensor measurements. The wires were connected to the impedance measurement circuit by means of silver conductive paint. The frequency of the ac current was varied from dc up to $2 \mathrm{MHz}$, but its magnitude was kept constant at $5 \mathrm{~mA}$ rms. A dc axial field was supplied by a pair of Helmholtz coils, in the range of \pm 100 Oe. Both the wire and Helmholtz coil axis were perpendicularly aligned with respect to the earth's magnetic field direction.

The axial induced voltage, $V_{z}$, is measured between the ends of the sample. A value of impedance is obtained through Ohm's law, and gives rise to the mostly reported MI effect, or so called axial magnetoimpedance.

In order to measure $V_{\phi}$, a pick-up coil ( $2.4 \mathrm{~cm}$ long, 1150 turns) was wounded on a glass tube of $1 \mathrm{~mm}$ of diameter and mounted around the wire. This voltage produces the offdiagonal impedance, as was explained above.

Generally, impedance in a wire is understood as a ratio of a voltage measured across it to a passing ac current, as shown in Fig. 1 [25], and the MI rate can then be defined with respect to the magnetic saturation of the sample at the maximum applied field $H_{\max }$ as

$\Delta Z / Z(\%)=100\left[Z(H)-Z\left(H_{\max }\right)\right] / Z\left(H_{\max }\right)$

where $Z$ is the impedance or one of its components $(Z=$ $\left.R+j X, j=(-1)^{1 / 2}\right)$. Here $Z$ can be the axial or the offdiagonal impedance.

Sensitivity to the applied magnetic field can be obtained from the impedance measurements [28] as

$s(\% / \mathrm{Oe})=2(\Delta Z / Z)_{\max } / \mathrm{FWHM}$

where FWHM is the full width at half maximum.

The influence of torsional stresses can also be investigated, instead of the applied magnetic field, due to the magnetoelastic coupling which produces changes in the magnetization processes of the samples without the influence of a magnetic field. A small change in the experimental setup shown in Fig. 1 can be made, consisting on the application of a torsional stress to the sample, without any magnetic field. The torsion-impedance ratio can be defined in exactly the same way as above, but with the application of torsional stresses, with respect to a maximum applied torsion $\xi_{\max }$, and without the influence of any magnetic field, as

$\Delta Z_{\xi} / Z(\%)=100\left[Z(\xi)-Z\left(\xi_{\max }\right)\right] / Z\left(\xi_{\max }\right)$

When the ac drive current, $I_{z}$, flows through a wire with helical magnetization, $I_{z}$ induces a voltage with a longitudinal and circular component, $V_{z}$ and $V_{\phi}$ respectively. $V_{z}$ is obtained between the clamped ends of the sample and $V_{\phi}$ from a pick-up coil wound around the wire. $I_{z}$ produces an ac circular magnetic field, $H_{\phi}$ (at the wire surface $H_{\phi}=I_{z} / 2 \pi a$, where $a$ is the wire radius), which is responsible for the change in the ac axial magnetization. Hence a circumferential or helical magnetization is needed in the sample to make it possible a cross-magnetization process.

\section{Experimental results}

The experimental procedure of producing the magnetic amorphous wires determines their peculiar domain structure, together with their magnetostriction coefficient. The domain structure of as-quenched ferromagnetic amorphous wires reflects the distribution of stress frozen-in during the fabrication process. Tensile, compressive and shear internal stresses are present in the samples and they depend on the distance to the wire's axis. The resultant magnetoelastic anisotropy together with the magnetostatic energy determines the magnetic regions present in the wire. Fe-rich wires have a positive (of the order of $10^{-5}$ ) magnetostriction coefficient, $\lambda_{s}$, and their domain structure consists on 


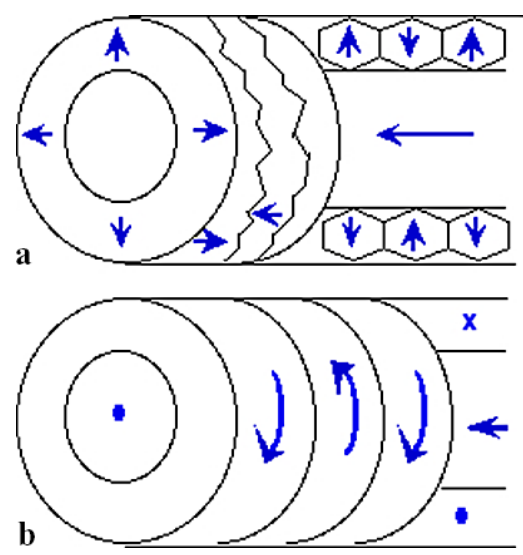

Fig. 2 Schematic representation of domain structure of Fe-rich $\left(\lambda_{s}>0\right),(\mathbf{a})$, and Co-rich $\left(\lambda_{s}<0\right),(\mathbf{b})$, amorphous wires in the as-quenched state

a longitudinally magnetized axial core, and a radially oriented outer shell, with a maze configuration and zig-zag walls on the whole surface, indicating the presence of stripe domains with unclosed [29] or closed magnetic flux, as can be observed in Fig. 2a. On the other hand the negative (of the order of $10^{-6}$ ) magnetostriction type wires (as e.g. Corich wires) also have the longitudinally oriented domain core but a circularly magnetized outer shell, separated by $180^{\circ}$ domain walls, as is displayed in Fig. $2 \mathrm{~b}$. There are also Co-based wires with vanishing magnetostriction coefficient with some weak straight walls and a few random helical domains with a bamboo-like structure that can hardly be observed at the surface, suggesting that the magnetization tends to align close to the circumferential direction.

The existence of the axially oriented core domain along the wire explains the magnetic bistability, consisting of the sudden reorientation of this inner domain [30]. Wires exhibiting vanishing magnetostriction coefficient do not show spontaneous magnetic bistability.

The thermal annealing of the wires and specially the application of torsional stresses or the annealing with an applied torsional stress changes the domain structure of the wires, changing also the magnetization processes that take place during the magnetization of the samples.

The skin effect appears when an AC current is applied to the wire [31]. The inner part of the sample is shielded to AC currents and fields, and the drive current flows through the outer shell of the wire. The measurements performed in our samples give us information of the magnetic response of their outer shell. How large this shell is, would be determined by other parameters that change the skin penetration depth, which can be roughly approximated by $\delta=$ $(\rho / \pi \mu f)^{1 / 2}$, being $\rho$ the electrical resistivity of the sample, $\mu$ the magnetic permeability and $f$ the drive current frequency. A change of these parameters would result in a different current distribution in the sample's cross section and thus a change in impedance. This approximation has given a reasonable qualitative agreement with experimental results [23]. The magnetic permeability depends as well on the drive current frequency in a complicated way, and is also affected by the magnetic anisotropy induced by the thermal treatment. In the case of nanostructured samples, $\rho$ changes with the thermal treatment, improving the impedance response with the nanocrystallization of the sample [25]. Therefore, in the case of high values of the drive current frequency, only the outer layer of the sample is responsible for the measured impedance, and for lower frequencies the axially magnetized inner domain would also intervene, if not all, at least some part of it.

\subsection{Frequency dependence of magnetoimpedance and torsion-impedance}

The impedance of the magnetic wires depends strongly on the drive current frequency used during the measurements. All measurements here presented were performed in the frequency range of $0.01-2 \mathrm{MHz}$, well below the ferromagnetic resonance observed in this kind of samples [32, 33]. In the frequency range of $0.1-10 \mathrm{MHz}$, either the bias-fieldinduced and/or the torsional-stress-induced changes in the sample's impedance reflects the variations of the magnetic penetration depth caused by strong changes in the transverse permeability in a soft magnetic conductor. At these frequencies, the driving current flows through the outer shell of the sample. The penetration depth $\delta$, depends on the transverse (with respect to the current direction) permeability of the sample $\mu_{t}$ as was explained above. The external dc field or torsional stress strongly modifies the permeability, leading to an increase in the effective section of the sample and a decrease in the impedance.

Figure 3 shows the torsion-impedance ratio spectra for $\mathrm{Fe}_{77.5} \mathrm{Si}_{7.5} \mathrm{~B}_{15}$ and $\mathrm{Co}_{72.5} \mathrm{Si}_{12.5} \mathrm{~B}_{15}$ wires that were measured in the as-quenched state and after being submitted to a torsional annealing treatment, as was explained above. The torsion-impedance shows an increase up to a maximum and then a decrease at higher driving current frequencies. The annealing treatment influences the torsion-impedance rate in a different way in each wire. In the positive magnetostrictive $\mathrm{Fe}_{77.5} \mathrm{Si}_{7.5} \mathrm{~B}_{15}$ wire the torsion-impedance response is improved by the annealing treatment. In the case of the negative magnetostrictive $\mathrm{Co}_{72.5} \mathrm{Si}_{12.5} \mathrm{~B}_{15}$ wire the torsion annealing treatment decreases the value of the maximum in the spectrum. This behaviour takes place in MI as well as TI spectra.

The drive current gives rise to a circular ac magnetic field that magnetizes the sample in the circular direction. The magnetization processes that take place are different according to the drive current frequency and to the torsional stress that modifies the domain structure. At low frequencies the magnetization processes take place by domain-wall 


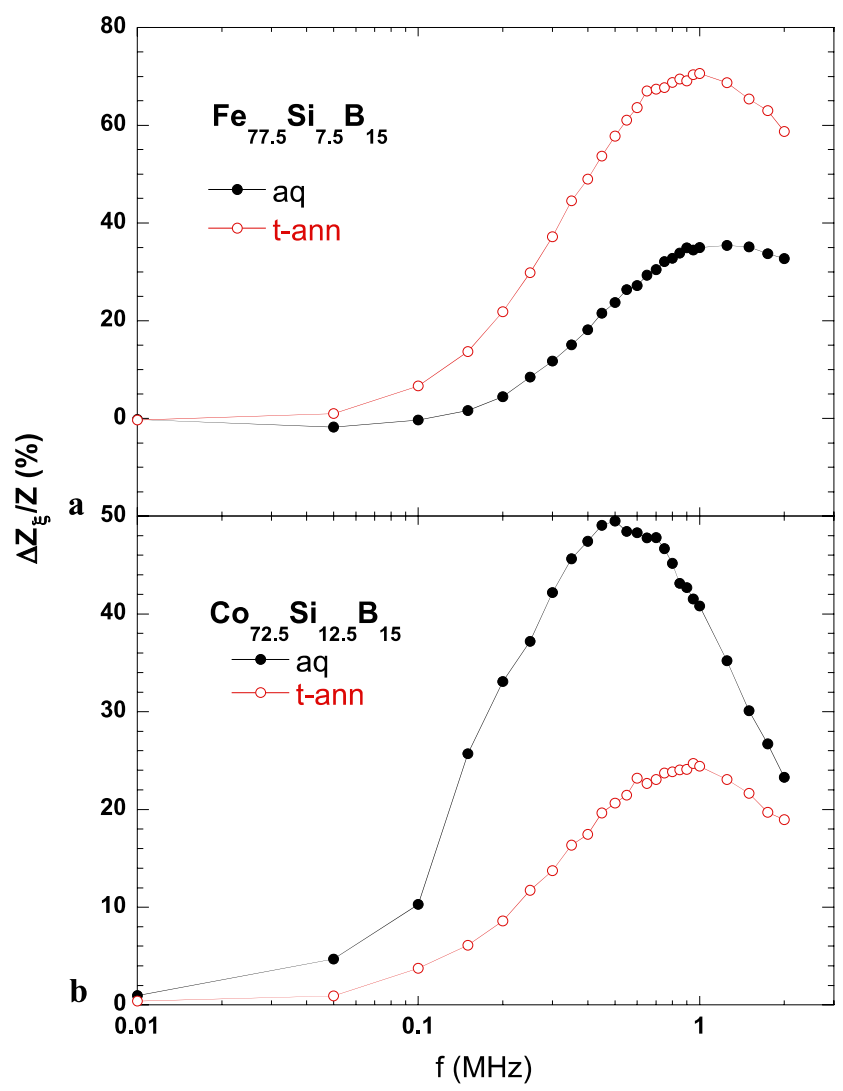

Fig. 3 Torsion-impedance ratio spectra for $\mathrm{Fe}_{77.5} \mathrm{Si}_{7.5} \mathrm{~B}_{15}$ (a) and $\mathrm{Co}_{72.5} \mathrm{Si}_{12.5} \mathrm{~B}_{15}$ (b) wires in the as-quenched state (aq), and after being submitted to an annealing treatment at $350^{\circ} \mathrm{C}$ during 20 min under the application of a torsional stress of $\pi / 20 \mathrm{rad} / \mathrm{cm},(\mathrm{t}-\mathrm{ann}) . I_{\mathrm{rms}}=5 \mathrm{~mA}$

displacements, and at larger frequencies, when the axial domain-wall movement has been damped, the influence of circular domains induced by the torsional stress is higher, resulting in a maximum in the torsion-impedance spectra. This maximum is characteristic for each sample, and it is called the relaxation frequency, $f_{x}$, separating two different magnetization processes that take place mainly at lower frequencies (domain-wall movements), from the ones at higher frequencies (magnetization rotation) [34].

The frequency at which the torsion-impedance has a maximum shows a small change with the annealing treatment in the case of the Fe-rich wire (from $1 \mathrm{MHz}$ to 1.25 MHz), and it increases from 0.5 MHz to $0.95 \mathrm{MHz}$ in the Co-rich wire for the as-quenched and torsion annealed samples, respectively.

The domain structure of the $\mathrm{Fe}_{77.5} \mathrm{Si}_{7.5} \mathrm{~B}_{15}$ and $\mathrm{Co}_{72.5} \mathrm{Si}_{12.5} \mathrm{~B}_{15}$ wires is quite different, due to the opposite sign in their magnetostriction coefficients. While the Fe-rich wire has an outer shell with radially oriented domains (see Fig. 2a), the Co-rich wire develops a circular domain structure in its outer shell, separated by $180^{\circ}$ domain walls as is shown in Fig. 2b. The application of a torsional stress during the annealing treatment would relax the stresses due to the fabrication procedure and would also induce a helical anisotropy. In the case of the $\mathrm{Fe}_{77.5} \mathrm{Si}_{7.5} \mathrm{~B}_{15}$ wire the positive sign of magnetostriction is essential in the development of the helical anisotropy due to its higher Curie temperature, as explained above. The helical induced anisotropy has the same direction of the applied torsional stress. The case of the $\mathrm{Co}_{72.5} \mathrm{Si}_{12.5} \mathrm{~B}_{15}$ wire is a little bit different, because the annealing treatment was performed above the Curie temperature, although the wire was cooled down in the presence of the applied torsional stress, and it develops a helical induced anisotropy as well, but with the helix angle perpendicular to the one of the applied torsional stress, due to its negative magnetostriction coefficient.

Figure 3 shows a higher value of $\Delta Z_{\xi} / Z(\%)$ maximum for the $\mathrm{Co}_{72.5} \mathrm{Si}_{12.5} \mathrm{~B}_{15}$ wire in the as-quenched state, because the circular domain structure favours the circular magnetization processes. When the wires are annealed, the $\mathrm{Fe}_{77.5} \mathrm{Si}_{7.5} \mathrm{~B}_{15}$ wire improves their magnetic behaviour, improving also the behaviour of the Co-rich wires in the asquenched state. The domain structure changed to a helical one, and the circular magnetization processes takes place by moment rotation. The application of a magnetic bias field (MI) instead of a torsional stress (TI) has a similar effect: there is a maximum of the spectra at a certain $f_{x}$ frequency, above which the magnetization processes are mainly due to moment rotation. This peculiar behaviour has also been found in ribbon-shaped samples [35].

\subsection{Influence of the induced anisotropy on the MI effect}

The magnetic field dependence of the MI gives information about the axial magnetization process. The domain-wall movements are irreversible and can produce some hysteresis in the magnetic field dependence of the impedance at low magnetic field values. The MI ratio versus $H$ curve shows the effect of a transverse anisotropy in the samples. For example, Fig. 4 shows the MI rate as a function of the applied bias field for $\mathrm{Fe}_{73.5} \mathrm{Si}_{13.5} \mathrm{~B}_{9} \mathrm{Nb}_{3} \mathrm{Cu}_{1}$ wires, with a diameter of $98 \mu \mathrm{m}$, in the as-quenched state and submitted to two different annealing treatments, one was a thermal annealing treatment at $560^{\circ} \mathrm{C}$ during 20 minutes in a furnace under $\mathrm{Ar}$ atmosphere (ann), and the second treatment was the same but with an applied torsional stress of $\pi / 20 \mathrm{rad} / \mathrm{cm}$ (t-ann). These measurements were performed at $1 \mathrm{MHz}$.

The as-quenched wire has a predominant easy magnetization wire axis, having a maximum of MI at nearly $H=$ 0 Oe field, and then decreasing for higher values of the applied field. The annealed samples show a typical two-peak structure due to the presence of a transverse anisotropy. In the case of the annealed sample (ann) the two peaks are very close to zero. In the torsion annealed (t-ann) sample the two peaks appear at a field of $\pm 10.7 \mathrm{Oe}$, revealing the existence of a transverse component of anisotropy. 


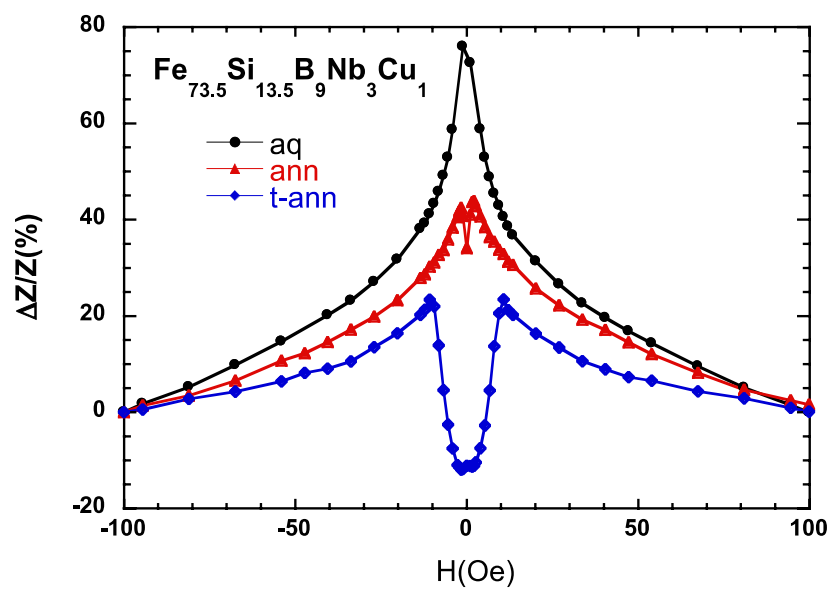

Fig. 4 MI rate as a function of the applied bias field for $\mathrm{Fe}_{73.5} \mathrm{Si}_{13.5} \mathrm{~B}_{9} \mathrm{Nb}_{3} \mathrm{Cu}_{1}$ wires: as-quenched $(\mathrm{aq})$, annealed at $560^{\circ} \mathrm{C}$ during $20 \mathrm{~min}(\mathrm{ann})$, and annealed at $560^{\circ} \mathrm{C}$ during $20 \mathrm{~min}$ with an applied torsional stress of $\pi / 20 \mathrm{rad} / \mathrm{cm}$ (t-ann). The driving current was $5 \mathrm{~mA}_{\mathrm{rms}}$ at $1 \mathrm{MHz}$

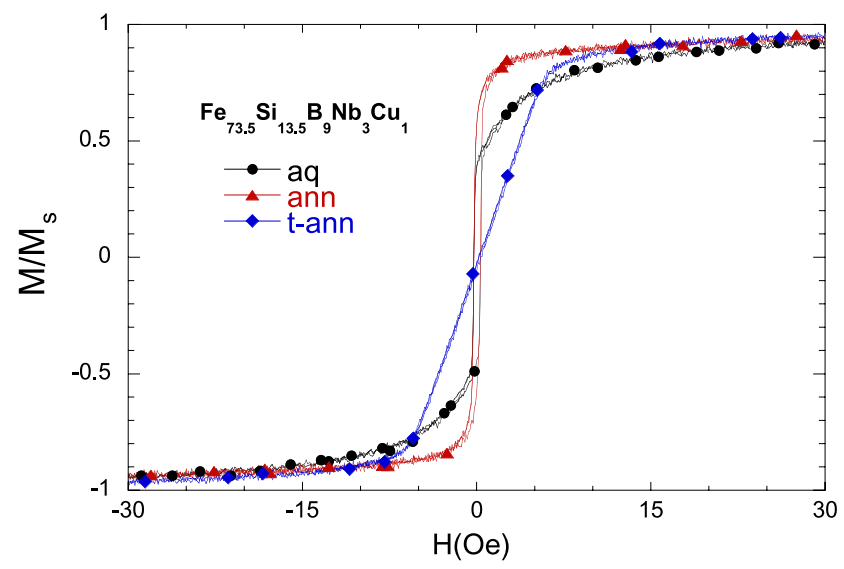

Fig. 5 Hysteresis loops of as-quenched (aq), annealed at $560^{\circ} \mathrm{C}$ during $20 \mathrm{~min}$ (ann), and annealed at $560^{\circ} \mathrm{C}$ during $20 \mathrm{~min}$ with an applied torsional stress of $\pi / 20 \mathrm{rad} / \mathrm{cm}$ (t-ann) $\mathrm{Fe}_{73.5} \mathrm{Si}_{13.5} \mathrm{~B}_{9} \mathrm{Nb}_{3} \mathrm{Cu}_{1}$ wires

The thermal annealing treatments produce a magnetic softening of the samples with respect to the wire in the as-quenched state, as can be seen from the values of the magnetostriction coefficients $\left(17.6 \times 10^{-6}, 4.5 \times 10^{-6}\right.$ and $\left.0.82 \times 10^{-6}\right)$ and the coercive fields $(0.44 \mathrm{Oe}, 0.23 \mathrm{Oe}$ and $0.06 \mathrm{Oe}$ ) for the as-quenched, annealed, and torsionalstress-annealed samples respectively. The magnetic softening of the sample would produce an increase in the magnetoimpedance response [21], but the existence of an induced transverse anisotropy also decreases this effect, due to the decrease of the magnetic permeability. In fact, both annealed wires exhibit a decrease in the MI response, as compared to the as-quenched state. The different induced anisotropies account for the MI behaviours. These induced anisotropies could be as the structural one [36] observed in stress-annealed amorphous Finemet-type alloys [37].

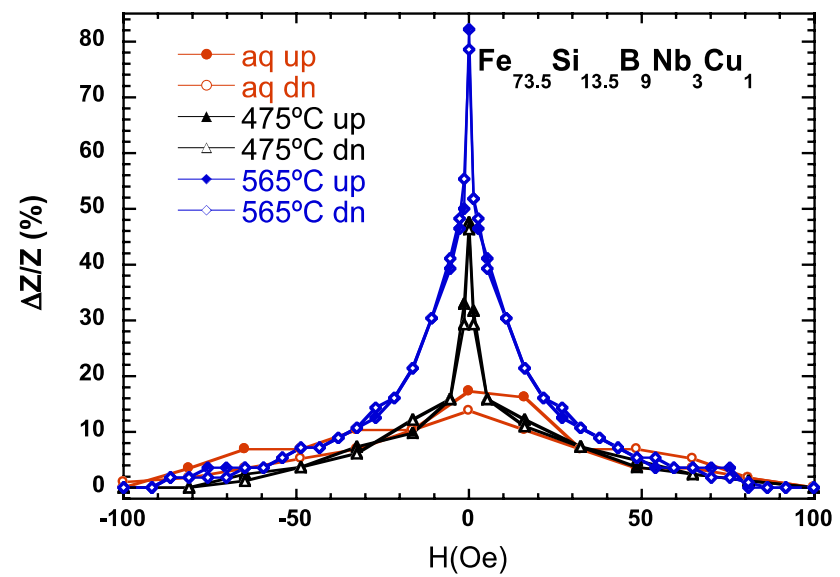

Fig. 6 MI effect in as-quenched (aq), and annealed $1 \mathrm{~h}$ at 475 and $565^{\circ} \mathrm{C} \mathrm{Fe}_{73.5} \mathrm{Si}_{13.5} \mathrm{~B}_{9} \mathrm{Nb}_{3} \mathrm{Cu}_{1}$ wires with $133 \mu \mathrm{m}$ diameter, as a function of the increasing (up) and decreasing (dn) applied magnetic field. $I_{\mathrm{rms}}=5 \mathrm{~mA}$ at $1,0.6$ and $0.25 \mathrm{MHz}$, respectively

The development of a transverse anisotropy in the torsion annealed (t-ann) wire can be clearly seen in the hysteresis loops of these samples, which are shown in Fig. 5, as well as the softening of the annealed (ann) wire, as compared to the as-quenched one. The magnetic MI response is not so large in the t-ann wire, due to the decrease of the magnetic permeability, clearly seen in this figure. The anisotropy determined from these hysteresis loops agrees with the position of MI peaks for this sample in Fig. 4.

\subsection{Microstructure influence on soft magnetic properties: nanocristallization}

The nanocrystallization process of amorphous $\mathrm{Fe}_{73.5} \mathrm{Si}_{13.5}$ $\mathrm{B}_{9} \mathrm{Nb}_{3} \mathrm{Cu}_{1}$ wires was studied obtaining the magnetization vs. temperature curves. This procedure allows us to see the crystallization temperatures of the different crystalline phases. The crystallization temperatures of these amorphous wires were around $520^{\circ} \mathrm{C}$ and $700^{\circ} \mathrm{C}$, respectively, and the Curie points of the different magnetic phases was around $350^{\circ} \mathrm{C}$ for the amorphous phase, around $620^{\circ} \mathrm{C}$ for the $\mathrm{FeSi}$ phase (with around 20 at. $\% \mathrm{Si}$ ), and around $400^{\circ} \mathrm{C}$ for the $\mathrm{Fe}_{2} \mathrm{~B}$ phase [38]. The annealing favours the soft magnetic response of the wires, giving rise to a high magnetic permeability and increasing the saturation magnetization.

Figure 6 shows the MI effect in three $\mathrm{Fe}_{73.5} \mathrm{Si}_{13.5} \mathrm{~B}_{9}$ $\mathrm{Nb}_{3} \mathrm{Cu}_{1}$ wires, with $133 \mu \mathrm{m}$ in diameter, one in the asquenched state and other two annealed during 1 hour at $475^{\circ} \mathrm{C}$ and $565^{\circ} \mathrm{C}$, at their relaxation frequencies, $1 \mathrm{MHz}$, $0.6 \mathrm{MHz}$ and $0.25 \mathrm{MHz}$ respectively. The maximum of MI appears at lower values, with the increasing treatment temperature, reflecting the structural changes that take place. The highest MI response takes place for the wire annealed at $565^{\circ} \mathrm{C}$, a selected temperature value that is near the primary crystallization temperature, when the wire has its soft- 


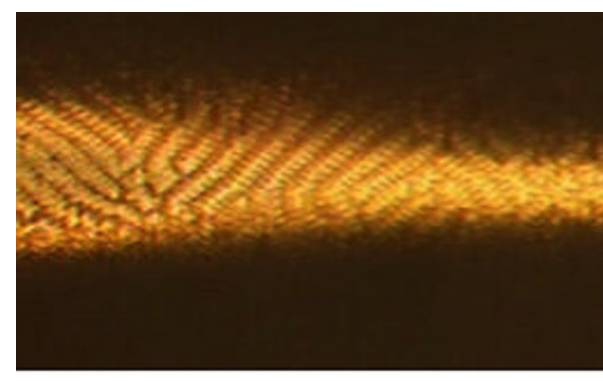

a

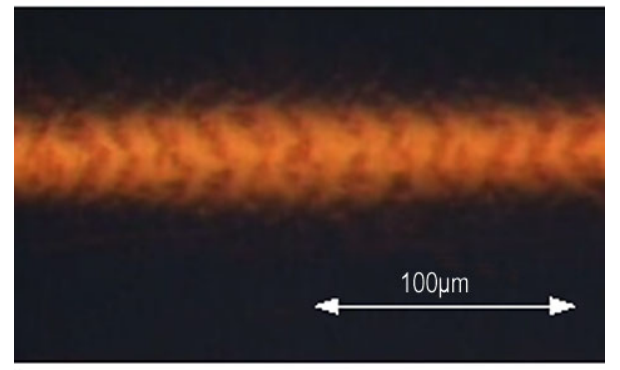

b

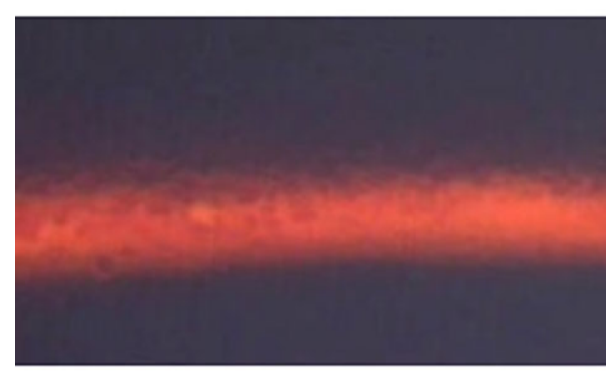

c

Fig. 7 Domain patterns of $\mathrm{Fe}_{73.5} \mathrm{Si}_{13.5} \mathrm{~B}_{9} \mathrm{Nb}_{3} \mathrm{Cu}_{1}$ wires with a $133 \mu \mathrm{m}$ diameter in the as-quenched (a) state and submitted to an annealing of $400^{\circ} \mathrm{C}(\mathbf{b})$ and $565^{\circ} \mathrm{C}(\mathbf{c})$ during 1 hour

est magnetic behaviour [39]. As it was explained above, the electrical resistivity affects the value of the skin penetration depth, decreasing it with respect to the non-nanocrystallized state [40]. In this figure the increasing and decreasing magnetic field effect is also shown, in order to observe a possible hysteretic behaviour. The as-quenched (aq) wire shows a small hysteresis at low values of the applied bias field. In the other annealed samples the hysteretic behaviour is negligible. This is also explained by the stress-relaxation effect due to the annealing treatments.

Besides, the MI effect is also sensitive to the effective anisotropy and the resulting domain structure. This fact can be observed by comparing the evolution of the MI effects and domain patterns revealed in Fig. 7. The disappearance for the labyrinthine domain pattern found on the as-quenched wire surface is detected. This effect is a consequence of the decrease in the average macroscopic anisotropy and the simultaneous reduction and compensation of the average magnetostriction.

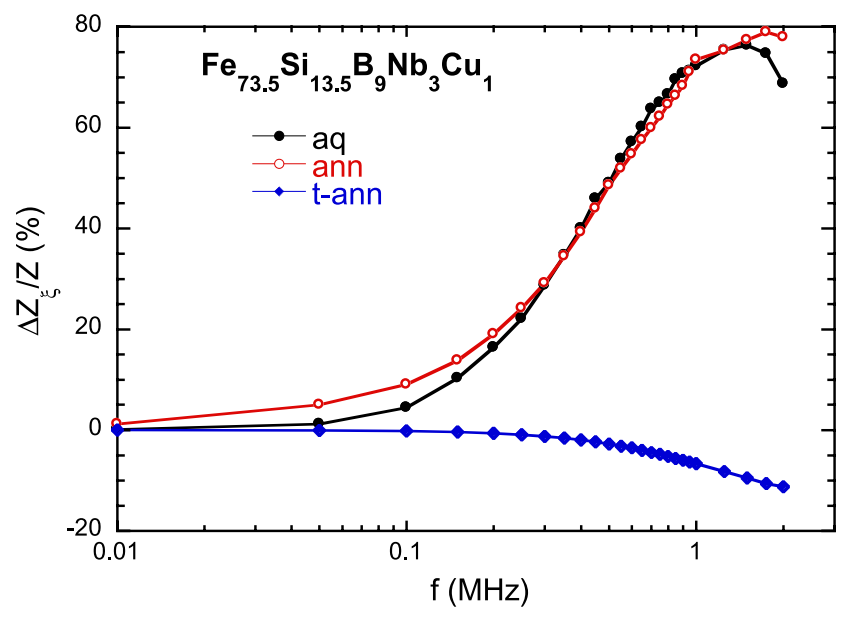

Fig. 8 TI rate spectra with respect to $1.26 \mathrm{rad} / \mathrm{cm}$ of the $\mathrm{FeSiBNbCu}$ wire in the as-cast (aq) state, relaxated (ann) and annealed with an applied torsional stress (t-ann)

\subsection{Torsion dependence: the TI effect}

Figure 8 shows the torsion-impedance (TI) rate spectra for the three $\mathrm{Fe}_{73.5} \mathrm{Si}_{13.5} \mathrm{~B}_{9} \mathrm{Nb}_{3} \mathrm{Cu}_{1}$ wires $(98 \mu \mathrm{m}$ diameter) subjected to different annealing treatments: as quenched, annealed at $560^{\circ} \mathrm{C}$ during 20 minutes (ann), and annealed at $560^{\circ} \mathrm{C}$ with an applied torsional stress of $\pi / 20 \mathrm{rad} / \mathrm{cm}$ (t-ann). The maximum values of the TI rate appear in the $\mathrm{MHz}$ range for all the cases. At a certain drive current frequency, the skin penetration depth, $\delta$, starts to be lower than the wire's radius and the application of a torsional stress, which changes the domain orientation towards a helical direction, modifies the local value of permeability, also changing $\delta$. As frequency increases, this effect takes place at more external shells of the wire, where the influence of a torsional stress is higher. It can be seen from Fig. 8 how the higher values appear in the case of the as-quenched wire and the one annealed without torsional stress. The application of a torsional stress in this Fe-rich wire decreases the value of the impedance response. The as-quenched and the relaxated wire show similar TI values at all frequency ranges. The annealing produces in the ann wire a relaxation of tensile stresses induced during the fabrication procedure. The response of the $\mathrm{t}$-ann wire, which was annealed with an applied torsional stress, is negative and very small. The nanocrystallization process in this wire took place under the influence of the applied torsional stress, which would induce a helical anisotropy in the wire. The impedance response is higher when the sample is twisted during measurement, resulting in a negative value of the torsional impedance (TI). It might be due to the nanocrystallization process, which favours the growth of $\mathrm{FeSi}$ crystallites and their orientation towards the circular direction is more difficult as they have been helically oriented. 


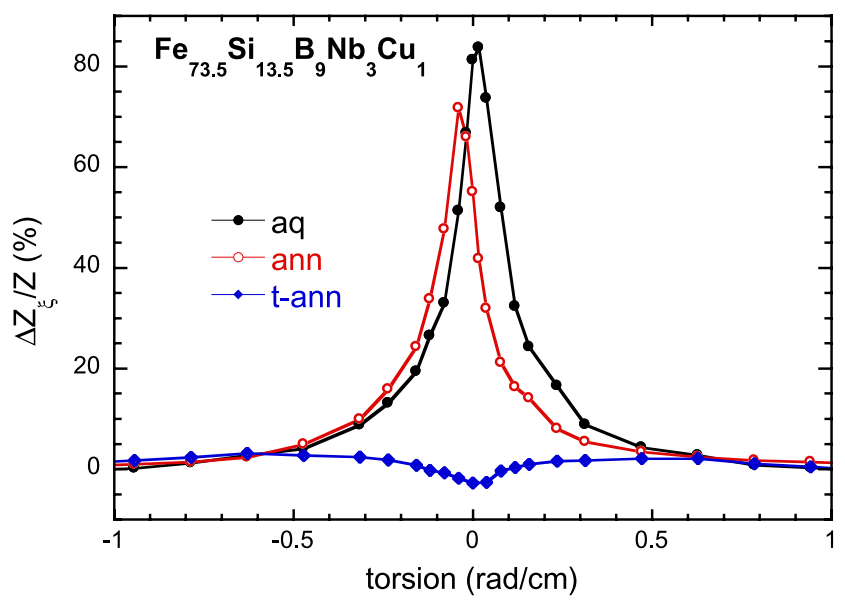

Fig. 9 Torsion Impedance of $\mathrm{FeSiBNbCu}$ as a function of the applied torsional stress for the as-quenched, and the annealed wires (aq, ann and t-ann). $I_{\mathrm{rms}}=5 \mathrm{~mA}$ at $1.5,1.5$ and $1.0 \mathrm{MHz}$, respectively

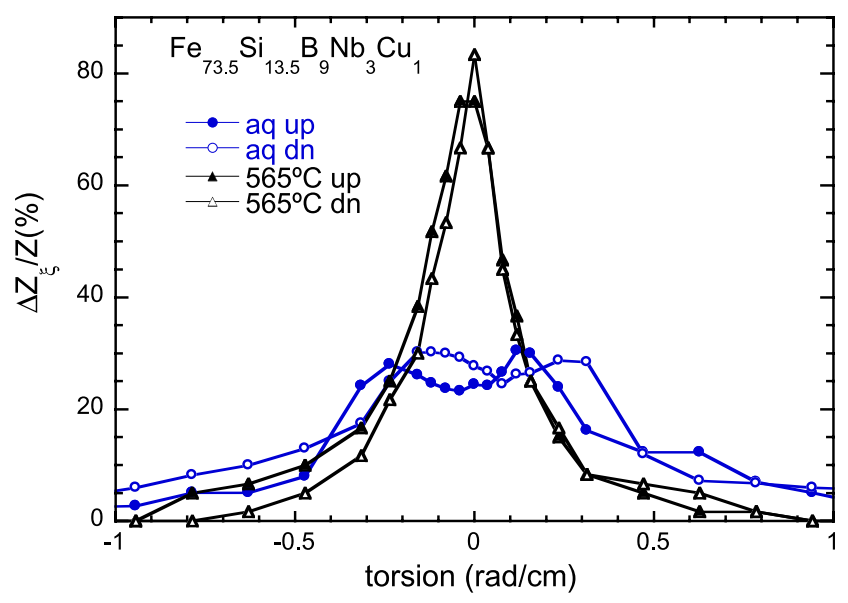

Fig. 10 The TI effect in $\mathrm{FeSiBNbCu}$ wires in their as-quenched state and after being annealed at $565^{\circ} \mathrm{C}$, at their relaxation frequencies of 0.9 and $0.2 \mathrm{MHz}$, respectively. $I_{\mathrm{rms}}=5 \mathrm{~mA}$

As it was explained above, a magnetic softening of the wires was obtained after the annealing treatment (ann), with the reduction of magnetostriction coefficient and the coercive field. But the magnetic permeability was also reduced in this wire, with the development of the helical anisotropy, which results in an anisotropy field of 10.7 Oe (see Fig. 4). The softening of the sample should improve the MI response, but the TI is a consequence of magnetoelastic effect, which should be larger for higher values of magnetostriction. This would explain the lower value in the case of the t-ann wire.

Figure 9 shows the TI rate as a function of the applied torsional stress. The effect is maximum around $\xi=0$, and decreases with increasing (clock wise) or decreasing (counter clock wise) applied torsional stress. Also, in this case the $\mathrm{t}$-ann wire shows a small response. The impedance change is more sensible to the application of a torsional stress in the aq and ann wires, due to the dominant of circular magnetizing effects created by the driving current with respect to the helical anisotropic t-ann wires [38, 41]. Figure 10 shows the TI effect for Fe wires (133 $\mu$ m diameter). It can be seen how the largest response is obtained in the case of the magnetically softest wire, annealed at $565^{\circ} \mathrm{C}$ at its relaxation frequency of $0.2 \mathrm{MHz}$. This can be ascribed to the strong reduction in the internal radial anisotropy when the wire is annealed at this temperature, as can be seen in Fig. 6 (domain structure). However, the TI response exhibits two peaks for the as-quenched wire at its relaxation frequency of $0.9 \mathrm{MHz}$, which can be ascribed to the internal anisotropy generated by the radial stress distribution responsible for the labyrinthine domain configuration. Some hysteresis with the increasing and decreasing torsional stress can be observed, especially in the as-quenched wire [38].

The impedance response of soft magnetic wires to the application of torsional stresses or magnetic field depends on the characteristics of the particular wire, like anisotropy or magnetostriction. The Fe-rich wires here studied in the as-quenched state show a larger response to the applied torsional stress, as compared with the conventional MI. The saturation magnetostriction in these wires is large, having a value of $\lambda_{S} \approx 35 \times 10^{-6}$ in $\mathrm{FeSiB}$ as-quenched wire and $18 \times 10^{-6}$ for the as-quenched $\mathrm{Fe}_{73.5} \mathrm{Si}_{13.5} \mathrm{~B}_{9} \mathrm{Nb}_{3} \mathrm{Cu}_{1}$, and they show $71 \%$ and $76 \%$ in the torsion impedance, which is much larger than the $17 \%$ and $54 \%$ shown in the MI response, respectively. When these wires are torsion annealed, a transverse helical anisotropy is developed, the domain structure is helically oriented in the outer shell of the wire, and the impedance response is not so sensible to the application of torsion during measurements. In these cases the field response shows larger values. A MI rate of $60 \%$ is obtained for the $\mathrm{FeSiB}$ annealed sample, which is larger than the $35 \%$ torsion-impedance response. In the case of this wire the saturation magnetostriction coefficient increased up to $42 \times 10^{-6}$, showing that the highest MI response is not always obtained in the lowest magnetostriction case.

In the case of Co-rich wires, the torsion annealing treatment changed magnetostriction from $-2.5 \times 10^{-6}$ to $-3 \times$ $10^{-6}$. The domain structure for the as-quenched wire is the one showed in Fig. 2b, while the annealing treatment favours the development of a helical outer-shell domain. The effect of torsion is not so large in these wires. TI of 50\% and $25 \%$ are obtained respectively (see Fig. 3), and the field response is better in both cases, with MI rates of $85 \%$ and $38 \%$, respectively.

The short annealing treatment in $\mathrm{Fe}_{73.5} \mathrm{Si}_{13.5} \mathrm{~B}_{9} \mathrm{Nb}_{3} \mathrm{Cu}_{1}$ wires at $560^{\circ} \mathrm{C}$ during 20 minutes produces the nanocrystallization of the samples. As it has been above mentioned, the as-quenched wire shows a larger impedance response to the applied torsion, but the nanocrystallized sample has a MI rate of $97 \%$, much larger than the TI rate of $79 \%$. The 


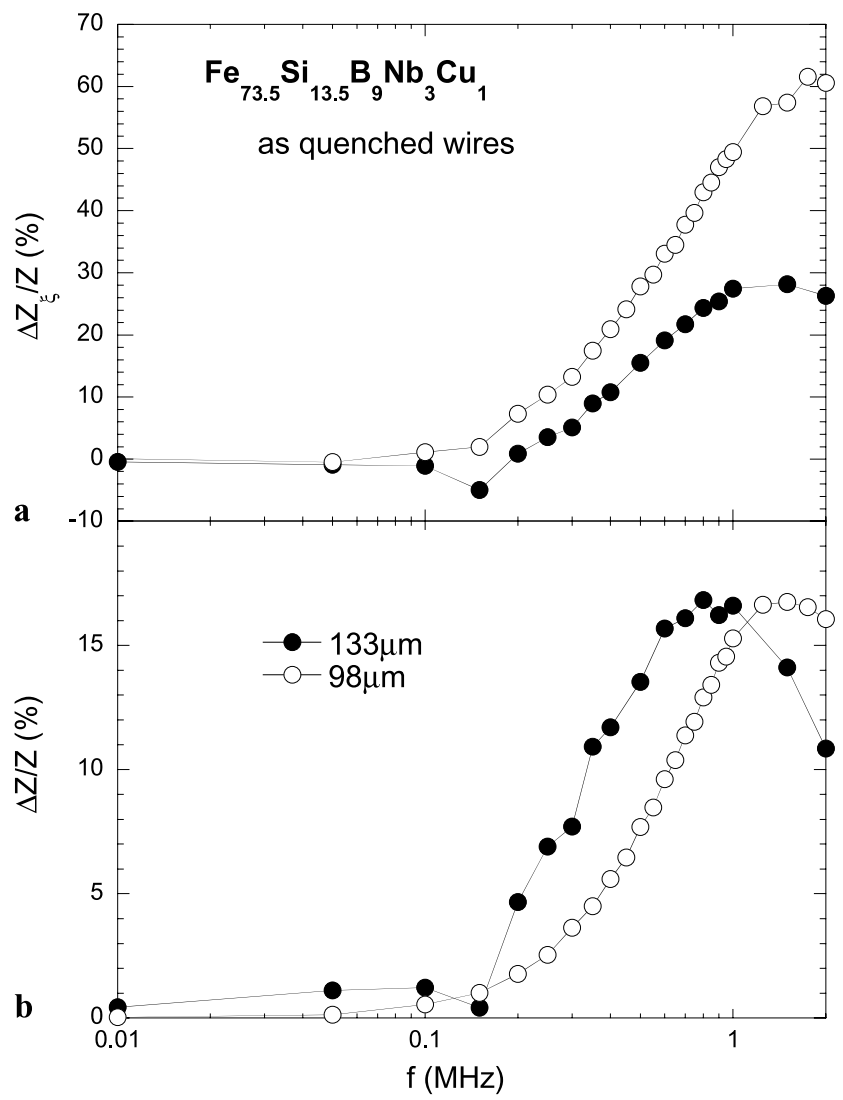

Fig. 11 Torsion-impedance rate with respect to $1.26 \mathrm{rad} / \mathrm{cm}$ (a) and magnetoimpedance rate (b) spectra for $\mathrm{FeSiBNbCu}$ wires with different diameters in their as-quenched state

appearance of small crystallites has magnetically softened the sample (magnetostriction decreased to $4.5 \times 10^{-6}$ ) and favours the TI and MI responses, as compared with the asquenched sample. This effect occurs as well in the $133 \mu \mathrm{m}$ diameter wires with this same composition, after an annealing of 1 hour in a furnace at $560^{\circ} \mathrm{C}$. In this case a TI rate of $83 \%$ and a MI rate of $82 \%$ are obtained, after the improving of the soft magnetic properties.

\subsection{Effect of the wire size on the impedance response}

Figure 11 shows the spectra of torsion impedance (a) and magnetoimpedance (b) of $\mathrm{FeSiBNbCu}$ wires in the asquenched state, having two different diameters: $133 \mu \mathrm{m}$ and $98 \mu \mathrm{m}$. The maximum applied torsional stress for Fig. 1a was $1.25 \mathrm{rad} / \mathrm{cm}$. The smaller $98 \mu \mathrm{m}$ diameter wire is more sensible to the application of torsional stresses in almost all the frequency range. The relaxation frequencies for the TI are similar in both cases. These wires are expected to have basically the same domain structure, but with variation of the proportion of volumes of the inner- and outer-shell domains. The application of torsional stresses affects the domain structure of the wires.

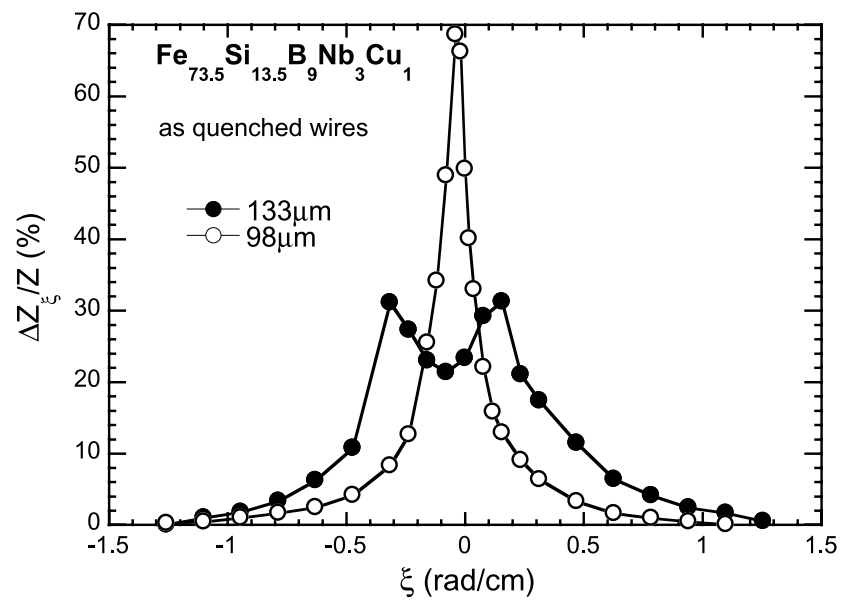

Fig. 12 Torsion impedance of FeSiBNbCu wires with different diameters for a drive current of $5 \mathrm{~mA}$ rms and $1.5 \mathrm{MHz}$

The response to the applied magnetic field (MI) of the bigger wire is larger in the frequency range up to $1 \mathrm{MHz}$, when the impedance of this wire decreased and the magnetoimpedance of the smaller wire is still increasing. The relaxation frequency increases as the wire diameter decreases, as happens in Co-rich glass covered wires [42]. The MI response as a function of the applied field is similar in both samples, with a single-peak structure, as is expected for the as-quenched wires. The different rate of the skin penetration depth to the wire radius value of both wires is not clearly seen in the starting point of the MI or TI effects.

The torsion impedance has a different character for both wires: there is a two-peak structure in the thicker wire, meanwhile the $98 \mu \mathrm{m}$ diameter wire shows a pronounced peak with the maximum at $\xi=0 \mathrm{rad} / \mathrm{cm}$, as is shown in Fig. 12 for both wires at $1.5 \mathrm{MHz}$. The smallest wire has the largest response to the applied torsional stress. As is expected in these wires, due to their large magnetostriction coefficient, the torsional-stress response is larger than the MI response (about 15\% in the $133 \mu \mathrm{m}$ diameter thick wire and $20 \%$ in the $98 \mu \mathrm{m}$ one, as compared with the $30 \%$ and $70 \%$ TI response). The different torsion response could be due to the influence of the domain structure of both wires. The torsion induces a helical anisotropy, which is strongest at the surface of the wire, therefore there could be still some inner domain axially oriented, and whose volume is larger in the thickest wire.

\subsection{Combined effect of magnetic field and applied torsional stress}

In order to study the influence of applied torsional stress on the MI effect, we measured the axial induced voltage in wires under the simultaneous application of torsions. Shear stresses introduced when a torque is applied on a rod are equivalent to the combination of tensile and compressive 


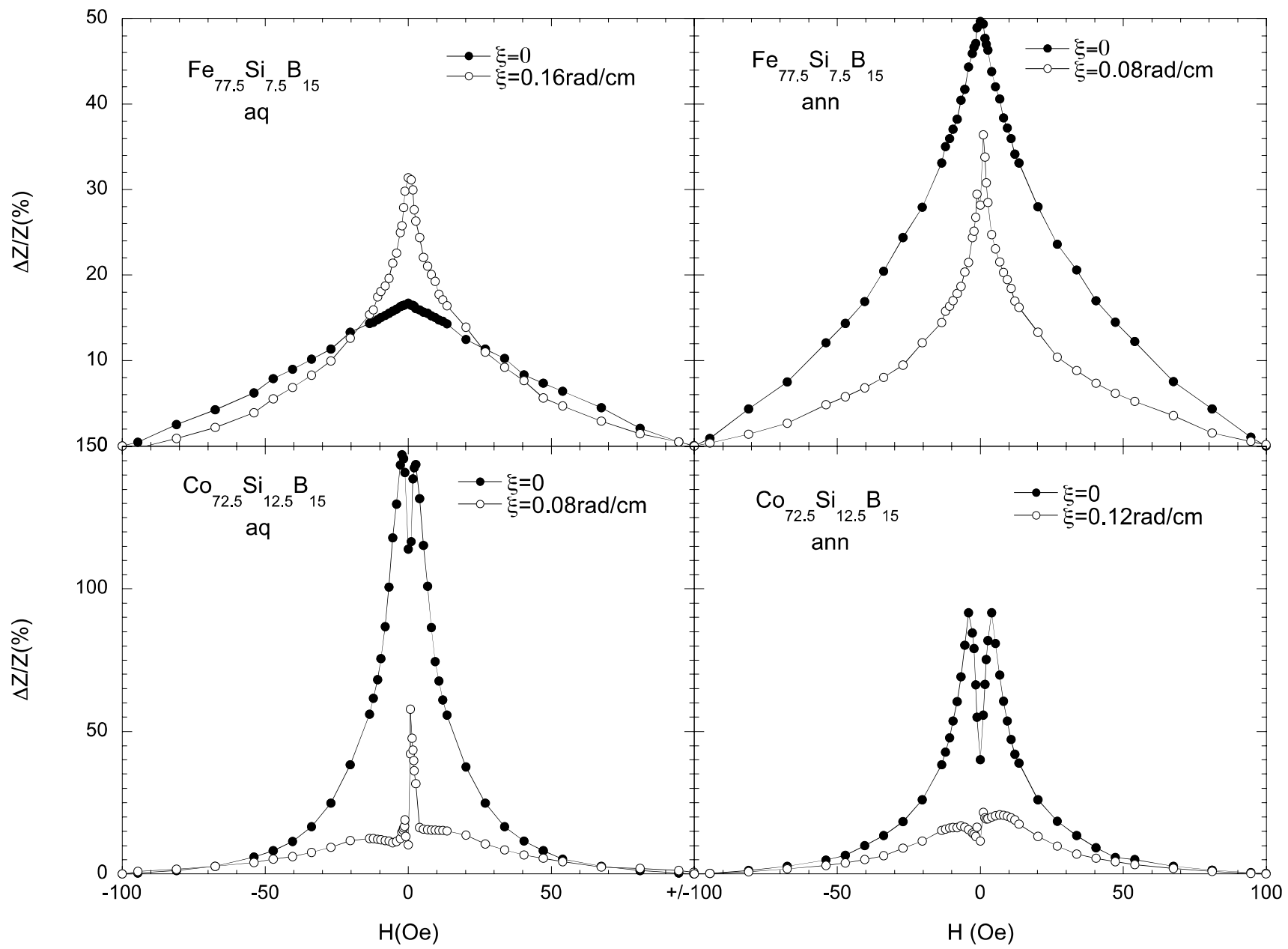

Fig. $13 \mathrm{MI}$ effect under the application of torsional stresses in $\mathrm{FeSiB}$ and $\mathrm{CoSiB}$ wires in the as-quenched (aq) state and annealed at $350^{\circ} \mathrm{C}$ during $20 \mathrm{~min}$ with a $\pi / 20 \mathrm{rad} / \mathrm{cm}$ torsion (ann). $I_{\mathrm{rms}}=5 \mathrm{~mA}$; the drive current frequencies are $0.8,0.6,0.5 \mathrm{and} 0.5 \mathrm{MHz}$, respectively

stresses of equal strength, mutually perpendicular and making an angle of $45^{\circ}$ with the rod's axis. That produces easy magnetoelastic directions following a helical path (tensile or compressive lines according to the sign of the saturation magnetostriction constant) [43].

Figure 13 shows the MI effect in some Fe-rich and Corich wires, in the as-quenched state and after being submitted to annealing treatments of $350^{\circ} \mathrm{C}$ with an applied tensile stress of $\pi / 20 \mathrm{rad} / \mathrm{cm}$. The selected value of driving current frequency was $0.8 \mathrm{MHz}$. The Co-rich wires have a larger field response with $\xi=0$ as is expected, reaching values close to $150 \%$ in the as-quenched state. The induction of helical anisotropy in all cases produces different responses. The as-quenched Fe-rich wire improves its response when a torsional stress is applied for the case of low magnetic field values $(H<12 \mathrm{Oe})$. This is due to the increase of the circular component of permeability, which improves the magnetization towards the axial direction as the magnetic $H$ field is increased. The annealed wires have already a helical anisotropy produced by the annealing treatment, but this reinforcement of the circular permeability would make more difficult the rotation of magnetization towards the axial direction. The impedance in the case of Fe-rich wires has better responses, but the application of an external torsional stress results in the decrease of the MI response.

The Co-rich wires show a two-peak structure, typical of samples with a circular anisotropy, when the maximum of permeability is obtained for a certain static magnetic field, where MI has a maximum [44]. In this case the application of torsional stresses largely decreases the MI response. The torsion applied to the samples during MI measurements favours the development of magnetic domains out of the circular direction and produces the tilting of the magnetization of the sample along the axial direction by means of rotational processes. The helical anisotropy would decrease the circular permeability that is initially present in the asquenched state, and is needed for a large MI effect.

Sensitivities to the applied magnetic field show their largest values in the case of the as-quenched Co-rich wire. The sensitivity value reaches $14.3 \%$ /Oe when no torsional 
stress is applied, but it is increased up to $19.1 \%$ /Oe with the application of $0.08 \mathrm{rad} / \mathrm{cm}$. This is due to the narrow peaks for this case, as can be observed in Fig. 13.

These results show that a small circular anisotropy is needed in order to obtain large impedance responses, although a further increase in anisotropy would lead to a decrease of permeability, and therefore a decrease of impedance.

\subsection{Off-diagonal MI in Fe-rich and Co-rich wires}

The off-diagonal MI effect originates in the existence of cross magnetization processes when the sample is axially magnetized and an ac current is flowing through it [22-25]. The large responses are favoured by the existence of a helical path around the direction of the excitation current [45].

The helical anisotropy that improves off-diagonal components of impedance was induced in our samples by the annealing treatments. Other authors develop a helical field by applying a dc current to the sample, together with the excitation current, in order to produce some kind of asymmetry. The dc current induces, in these cases, a completely different appearance of the off-diagonal MI. The handicap of this latter procedure is that a higher consumption is needed [22, $23,46]$. The same asymmetry found in off-diagonal components, has been also found in axial impedance by the effect of dipolar interactions between two different samples [47].

The off-diagonal impedance dependence on axial field is shown in Figs. 14, 15 and 16, for a variety of Fe-rich and Co-rich wires, measured at their relaxation frequencies, where the maximum response to the $H$ field is obtained. The off-diagonal MI response improves with the annealing treatments for all measured wires. The case of the $\mathrm{FeSiB}$ wires is displayed in Fig. 14. The annealing treatment makes the offdiagonal MI sharper and improves sensitivity up to $16 \%$ /Oe. Although the magnetostriction increases up to $42 \times 10^{-6}$, a helical anisotropy is developed, and it is stronger on the wire's surface, increasing the circular permeability, as compared with the as-quenched wire.

The case of $\mathrm{CoSiB}$ wires, with negative magnetostriction can be observed in Fig. 15. The annealing treatment largely improves the off-diagonal impedance response, reaching values up to $3300 \%$. The sensitivities are huge: $106 \% / \mathrm{Oe}$ in the as-quenched wire, and $600 \% / \mathrm{Oe}$ in the annealed one. The two-peak MI behaviour that appears for these wires has been related to the rotational magnetization process occurring near the anisotropy field of the circular domain in the outer shell of the wire $[27,48]$. The circular anisotropy field is then \pm 2 Oe for the as-quenched wire and decreases a little bit, to \pm 1.5 Oe in the annealed one. This shows that the circular permeability decreases due to the development of the helical induced anisotropy. The off-diagonal $\zeta_{\phi z}$ component has been enhanced for the reinforced helical anisotropy at the wire surface $[49,50]$.

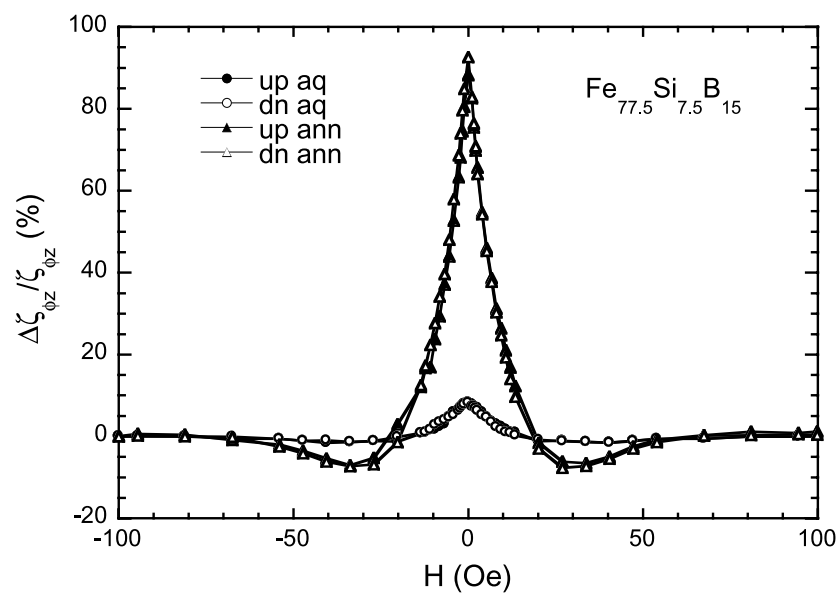

Fig. 14 Off-diagonal component of magnetoimpedance for Fe-rich wires in the as-quenched (aq) state and after being submitted to a $350^{\circ} \mathrm{C}$ annealing with a torsional stress of $\pi / 20 \mathrm{rad} / \mathrm{cm}$ (ann) at 0.8 and $0.6 \mathrm{MHz}$, respectively

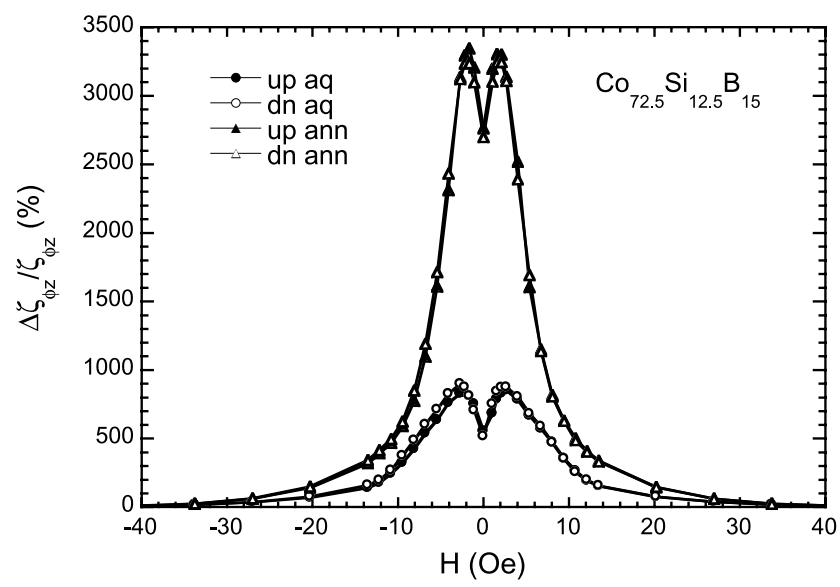

Fig. 15 Off-diagonal component of magnetoimpedance for Co-rich wires in the as-quenched (aq) state and after being submitted to a $350^{\circ} \mathrm{C}$ annealing with a torsional stress of $\pi / 20 \mathrm{rad} / \mathrm{cm}$ (ann) at $0.45 \mathrm{MHz}$

Figure 16 shows the case of other $\mathrm{Fe}_{73.5} \mathrm{Si}_{13.5} \mathrm{~B}_{9} \mathrm{Nb}_{3} \mathrm{Cu}_{1}$ wires, in the aq state, after being partially nanocrystallized (ann), and the last ones after being torsion annealed (t-ann), as they were above defined. The torsion annealing treatment increases the off-diagonal MI response up to $720 \%$, and develops a very well defined structure of two peaks, meanwhile the aq and ann wires have a single-peak structure. This has been related to the development of the helical anisotropy induced by the annealing treatment with the applied torsional stress. This result seems to indicate that after the annealing treatment, the torsion-induced helical anisotropy has partially balanced the intrinsic one, decreasing the angle between the easy axis and the wire axis. Although the helical anisotropy is not uniform along the wire radius, its magnitude is the highest at the wire surface changing the domain structure $[27,51]$. 
Fig. 16 Off-diagonal

component of

magnetoimpedance in Fe-rich wires in the as-quenched state (aq), after being submitted to an annealing treatment of $560^{\circ} \mathrm{C}$ (ann), and the same treatment with a torsional stress $\left(560^{\circ} \mathrm{C}\right.$, $\pi / 20 \mathrm{rad} / \mathrm{cm}$ ), (t-ann). The relaxation frequencies are 1.5 , 1.5 and $1.0 \mathrm{MHz}$, respectively

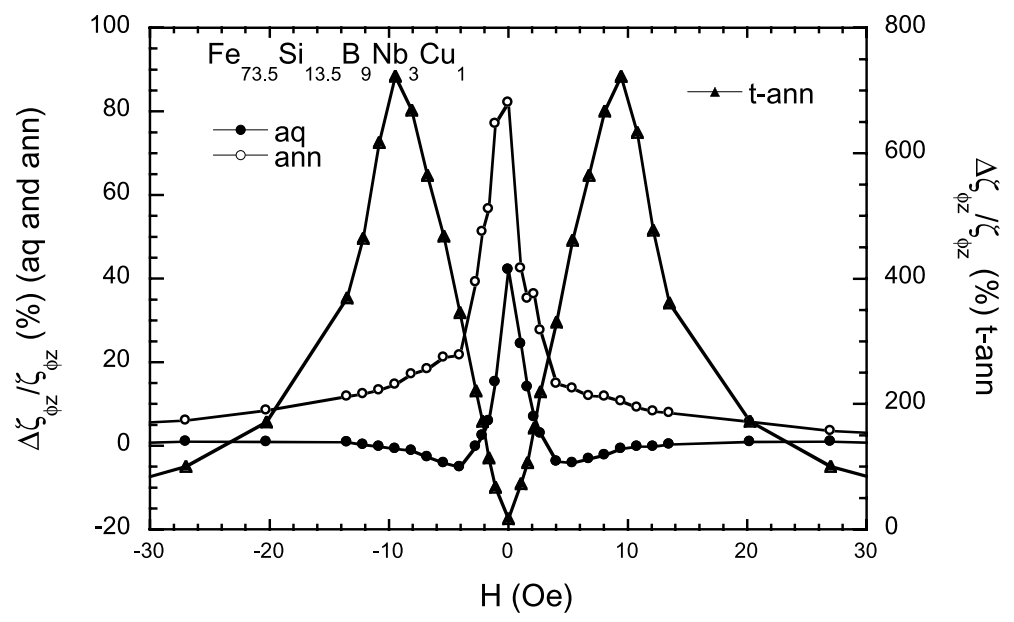

All materials studied in this work improve their offdiagonal impedance response when the helical anisotropy develops. All results show almost symmetrical curves with no appreciable hysteresis due to the fact that no dc bias current has been applied during measurements. The application of a dc bias current has been demonstrated to increase the asymmetry in the low field region [52]. This has the advantage of reducing the power consumption when a sensor based on this effect is built, but it reduces the linear part of the curve from zero up to the anisotropy field value.

The quick decrease in the off-diagonal component of impedance is due to the decreasing of circular permeability, which takes place as the axial magnetizing field increases. The lack of hysteresis suggests that the magnetization process takes place by reversible rotational processes from the helical to the axial direction. The high frequency also guarantees the absence of domain-wall displacements due to their damping.

The huge sensitivity obtained with this effect could be influenced by the small value of induced voltage at the maximum applied field, which is used to calculate the sensitivity from (2) and (3). This makes possible the use of these samples as sensitive elements in magnetic field sensors based on this effect. For example in Fig. 15, the magnetic fields between +2 Oe and up to 20 Oe could be easily measured from this strong dependence, using the more sensitive annealed Co-rich sample as sensing element. The behaviour of the annealed Fe-rich wire in Fig. 14 would allow us to detect magnetic fields from 0 Oe up to around 20 Oe in the quick decrease of the off-diagonal component of impedance with a smaller sensitivity than the Co-rich wire.

\section{Conclusions}

Large varieties of amorphous wires have been studied in this work, in order to obtain the axial and off-diagonal impedance response to magnetic field and/or torsional stresses, and under the influence of different stress/torsion-induced anisotropies and annealing treatments for samples nanocrystallization.

The different MI behaviour in wires is mainly due to essential differences in their domain structure due to their opposite sign of magnetostriction coefficients (Co-rich and Ferich wires) or to the induced anisotropies, which change the circular magnetic permeability, influencing the magnetic response of the material.

The different diameter of the wires also modifies the proportion of the inner and outer domains, producing different MI and TI responses.

The simultaneous application of magnetic fields and torsional stresses can improve the magnetic response of impedance in some of the wires, due to the development of the magnetoelastic helical anisotropy modifying the outer-shell magnetic domain structure, which produces responses to the applied field of $150 \%$ in the most favourable situation of the Co-rich wire. A small circular anisotropy is desirable in order to obtain large responses.

The off-diagonal component of impedance has been studied in amorphous and nanocrystallized wires. The offdiagonal MI effect response is found to be due to the decreasing of circular permeability when the samples are axially magnetized, increasing the magnetization processes by reversible rotation of magnetization. This effect seems to have several advantages for its application in magnetic field sensing devices. This component shows enormous almost symmetrical responses to the applied magnetic field and their responses do not exhibit hysteresis.

The off-diagonal MI responses improve with the development of a helically induced anisotropy in torsion annealed samples. The nanocrystallization of the sample wires also improves the off-diagonal effect, due to the magnetic softening of the samples.

Although the off-diagonal MI effect is not asymmetric around $H=0 \mathrm{Oe}$, its large value makes it possible for using 
it in the measurement of magnetic fields without the application of any extra dc current, which would increase the power consumption of the sensing device, allowing its noise to decrease at the same time.

Acknowledgements This work has been supported by the Spanish MEC under the research project MAT2009-13108-C02-01 and FICYT under the research project IB09-131.

\section{References}

1. L.V. Panina, K. Mohri, Appl. Phys. Lett. 65, 1185 (1994)

2. M. Vázquez, M. Knobel, M.L. Sánchez, R. Valenzuela, A.P. Zhukov, Sens. Actuators A 59, 20 (1997)

3. A.P. Zhukov, J. González, J.M. Blanco, M. Vazquez, V.S. Larin, J. Mater. Res. 15, 2107 (2000)

4. M. Knobel, M. Vázquez, L. Kraus, in Giant Magnetoimpedance, ed. by K.J.H. Buschow. Handbook of Magnetic Materials, (Elsevier, New York, 2002)

5. K. Mohri, T. Uchiyama, L.P. Shen, C.M. Cai, L.V. Panina, Y. Honkura, M. Yamamoto, IEEE Trans. Magn. 38, 3063 (2002)

6. D.C. Jiles, Acta Mater. 51, 5907 (2003)

7. M. Knobel, K.R. Pirota, J. Magn. Magn. Mater. 242, 33 (2002)

8. N.A. Usov, A.S. Antonov, A.N. Lagar'kov, J. Magn. Magn. Mater. 185, 159 (1998)

9. V.M. Prida, M.L. Sánchez, B. Hernando, P. Gorria, M. Tejedor, M. Vazquez, Appl. Phys. A, Mater. Sci. Process. 77, 135 (2003)

10. M. Tejedor, B. Hernando, M.L. Sánchez, V.M. Prida, M. Vázquez, J. Phys. D, Appl. Phys. 31, 3331 (1998)

11. M. Tejedor, B. Hernando, M.L. Sanchez, V.M. Prida, M. Vazquez, Sens. Actuators A 81, 98 (2000)

12. J.M. Blanco, A.P. Zhukov, J. González, J. Phys. D, Appl. Phys. 87, 4813 (2000)

13. T.A. Furmanova, A.S. Antonov, I.N. Morozov, L.V. Panina, Sens. Actuators A 81, 95 (2000)

14. I. Betancourt, R. Valenzuela, Appl. Phys. Lett. 81, 94 (2002)

15. Y.F. Li, M. Vazquez, D.X. Chen, J. Appl. Phys. 93, 9839 (2003)

16. B. Hernando, V.M. Prida, M.L. Sánchez, P. Gorria, G.V. Kurlyandskaya, M. Tejedor, M. Vazquez, J. Magn. Magn. Mater. 258-259, 183 (2003)

17. A. Zhukov, J. Gonzalez, in Advanced Magnetic Materials, vol. 3, ed. by Y. Liu, D.J. Sellmyer, D. Shindo (Kluwer Academic, Dordrecht, 2006), Chap. 5

18. G. Herzer, Scr. Metall. Mater. 33, 1741 (1995)

19. M.E. McHenry, M.A. Willard, D.E. Laughlin, Prog. Mater. Sci. 44, 291 (1999)

20. K. Suzuki, G. Herzer, in Advanced Magnetic Nanostructures, ed. by D.J. Sellmyer, R. Skomski (Kluwer Academic, Dordrecht, 2005), Chap. 13

21. B. Hernando, P. Gorria, M.L. Sanchez, V.M. Prida, G.V. Kurlyandskaya, in Encyclopedia of Nanoscience and Nanotechnology, vol. 4, ed. by H.S. Nalwa (ASP, Syracuse, 2004), p. 949

22. M. Ipatov, V. Zhukova, J.M. Blanco, J. González, A. Zhukov, Phys. Status Solidi A 205, 1779 (2008)

23. D.P. Makhnovskiy, L.V. Panina, D.J. Mapps, Phys. Rev. B 63, 144424 (2001)
24. V.M. Prida, P. Gorría, G.V. Kurlyandskaya, M.L. Sánchez, B. Hernando, M. Tejedor, Nanotechnology 14, 231 (2003)

25. S. Sandacci, D.P. Makhnovskiy, L.V. Panina, K. Mohri, Y. Honkura, IEEE Trans. Magn. 40, 3505 (2004)

26. A. Hernando, Phys. Scr. T 24, 11 (1998)

27. T. Sánchez, P. Alvarez, J. Olivera, M.J. Pérez, F.J. Belzunce, J.D. Santos, J.L. Sánchez Ll, M.L. Sánchez, P. Gorría, B. Hernando, J.'Non-Cryst. Solids 353, 914 (2007)

28. R.S. Beach, A.E. Berkowitz, Appl. Phys. Lett. 64, 3652 (1994)

29. Yu. Kabanov, A. Zhukov, V. Zhukova, J. González, Appl. Phys. Lett. 87, 142507 (2005)

30. K. Mohri, F.B. Humphrey, K. Kawashima, K. Kimura, M. Mizutani, IEEE Trans. Magn. 26, 1789 (1990)

31. L. Chen, Y. Zhou, C. Lei, Z.-M. Zhou, W. Ding, Phys. Status Solidi A 448 (2010)

32. M.R. Britel, D. Menard, L.G. Melo, P. Ciureanu, A. Yelon, R.W. Cochrane, M. Ouabhi, B. Cornut, Appl. Phys. Lett. 77, 2737 (2000)

33. L.V. Panina, D.P. Makhnovskiy, K. Mohri, J. Magn. Magn. Mater. 272-276, 1452 (2004)

34. M.L. Sánchez, R. Valenzuela, M. Vázquez, A. Hernando, J. Mater. Res. 11, 2486 (1996)

35. B. Hernando, M.L. Sánchez, V.M. Prida, M. Tejedor, M. Vázquez, J. Appl. Phys. 90, 4783 (2001)

36. Y. Suzuki, J. Khaimovich, T. Egami, Phys. Rev. B 35, 2162 (1987)

37. C. Miguel, A. Zhukov, J. González, Phys. Status Solidi A 194, 291 (2002)

38. B. Hernando, J. Olivera, M.L. Sánchez, V.M. Prida, M.J. Perez, J.D. Santos, P. Gorría, F.J. Belzunce, Phys. Met. Metallogr. 102, S13 (2006)

39. P. Gorria, V.M. Prida, M. Tejedor, B. Hernando, M.L. Sánchez, Physica B 299, 215 (2001)

40. M. Knobel, M.L. Sánchez, P. Marín, C. Gómez-Polo, M. Vázquez, A. Hernando, IEEE Trans. Magn. 31, 4009 (1995)

41. B. Hernando, V.M. Prida, M.L. Sánchez, J. Magn. Magn. Mater. 258, 183 (2003)

42. D. García, V. Raposo, J.I. Iñiguez, O. Montero, M. Zazo, J. Magn. Magn. Mater. 290, 1078 (2005)

43. L. Kraus, S.N. Kane, M. Vázquez, G. Rivero, E. Fraga, A. Hernando, J.M. Barandiarán, J. Appl. Phys. 75, 6952 (1994)

44. M. Vázquez, J. Magn. Magn. Mater. 226, 693 (2001)

45. M. Malátek, L. Krauss, Sens. Actuators A 164, 41 (2010)

46. L. Krauss, J. Magn. Magn. Mater. 320, e746 (2008)

47. J.P. Sinnecker, A.E.P. de Araujo, R. Piccin, M. Knobel, M. Vázquez, J. Magn. Magn. Mater. 295, 121 (2005)

48. M. Knobel, M. Vázquez, L. Krauss, in Giant Magnetoimpedance, ed. by K.J.H. Buschow. Handbook of Magnetism and Magnetic Materials, vol. 12 (Elsevier, New York, 2003), p. 497

49. A.P. Chen, C. García, A. Zhukov, L. Domínguez, J.M. Blanco, J. González, J. Phys. D, Appl. Phys. 39, 1718 (2006)

50. N.A. Buznikov, A.S. Antonov, A.B. Granovsky, C.G. Kim, C.O. Kim, X.P. Li, S.S. Yoon, J. Magn. Magn. Mater. 296, 77 (2006)

51. D.X. Chen, L. Pascual, F.J. Castaño, M. Vázquez, A. Hernando, IEEE Trans. Magn. 37, 994 (2001)

52. N.A. Buznikov, C.G. Kim, C.O. Kim, S.S. Yoon, J. Magn. Magn. Mater. 309, 216 (2007) 